\title{
ON DYNAMICS OF CERTAIN ECONOMIC MODELS
}

The article deals with the dynamics of two economic models. The first one is a macroeconomic model about national income and its stability. In the second model the price of a certain commodity is treated. The equilibrium points, periodic points, price cycles and their stability in the market is also observed. The article is interdisciplinary in its nature.

Keywords: Economic model, national income, price, equilibrium point, periodic point, cycle, stability.

\section{Introduction}

The basic dynamical macroeconomics model for national income $Y$ follows from the equilibrium condition

$$
Y=C+I
$$

where $C$ is national consumption and $I$ is national investment. The usual dynamical assumption is that consumption depends on income with a one-period lag, i.e.

$$
C(t)=a+b Y(t-1), a \geq 0,0<b<1,
$$

where the constant $a$ characterizes consumption, $b$ is the marginal propensity to consume and $t$ is time. The investment is assumed to be partly autonomous and in the initial period shifts from $I_{0}$ to $I_{0}+\Delta I$ and remaining at this level in all subsequent periods and partly depending on income (with a one-period lag) according to the marginal propensity to invest $h$ :

$$
I(t)=I_{0}+\Delta I+h Y(t-1), 0<h<1 .
$$

Then the difference equation

$$
Y(t)=C(t)+I(t)
$$

is interpretation of the model.

Our aim is to investigate the stability of the model (2) which expresses its dynamics in the case when the investment has a form

$$
\begin{aligned}
& I(t)=h Y(t-1)+\left(I_{0}+\Delta I\right)\left(1-d^{t}\right), \\
& 0<h<1,0 \leq d \leq 1 .
\end{aligned}
$$

The value $I_{0}+\Delta I$ does not remain constant in all the subsequent periods.

From the mathematical point of view we also study the dynamics of the pricing of a certain commodity. In this model our attention is focused on the equilibrium points, periodic points, cycles and their stability.

\section{The basic model of national income}

We consider the case when $0<d<1$ in (3). We invest it gradually. We remind that if $d=0$ we obtain the model from [3]. If $d=1$ then the investment depends only on the national product $Y(t-1)$.

So, in the case $0<d<1$ the part of investment

$$
\left(I_{0}+\Delta I\right)\left(1-d^{t}\right)
$$

will gradually increase from the zero value in the time $t=0$ to the value $I_{0}+\Delta I$ as $t \rightarrow \infty$.

Applying (1) and (3) in (2) we get

$$
\begin{aligned}
& Y(t)-(b+h) Y(t-1)=a+I_{0}+\Delta I-\left(I_{0}+\Delta I\right) d^{t}, \\
& t \geq 0
\end{aligned}
$$

The difference equation (4) represents the model (2).

For the simplicity we assume that $d \neq b+h \neq 1$. We can find the particular solution $Y^{*}(t)$ of (4) in the form

$$
Y^{*}(t)=Y_{1}^{*}(t)+Y_{2}^{*}(t),
$$

where $Y_{1}^{*}(t)$ or $Y_{2}^{*}(t)$ is a particular solution of the equation

$$
Y(t)-(b+h) Y(t-1)=a+I_{0}+\Delta I
$$

or

$$
Y(t)-(b+h) Y(t-1)=-\left(I_{0}+\Delta I\right) d^{t} .
$$

The general solution of the difference equation (4) has a form $Y(t)=\bar{Y}(t)+Y_{1}^{*}(t)+Y_{2}^{*}(t)$,

\section{* Rudolf Olach, Lubica Hanuštiaková}

Department of mathematical analysis and applied mathematics, Faculty of natural sciences, University of Zilina,

E-mail: rudolf.olach@fpv.utc.sk, lubica.hanustiakova@fpv.utc.sk 
where $\bar{Y}(t)$ is the general solution of the homogeneous part of (4), i. e.

$$
Y(t)-(b+h) Y(t-1)=0 .
$$

The characteristic equation of (7) is $\lambda-(b+h)=0$. Then the general solution of (7) becomes

$$
\bar{Y}(t)=A \lambda^{t}=A(b+h)^{t}, \quad A \in R, \quad t \geq 0,
$$

The particular solution $Y_{1}^{*}(t)$ of (5) with assumption $b+h \neq 1$ has a form

$$
Y_{1}^{*}(t)=A_{1}, \quad A_{1} \in R
$$

and applying in (5) we get $A_{1}=\left(a+I_{0}+\Delta I\right) /(1-b-h)$. So, we have

$$
Y_{1}^{*}(t)=\frac{a+I_{0}+\Delta I}{1-b-h} .
$$

Now we will find the particular solution of (6) in the form $Y_{2}^{*}(t)=A_{2} d^{t}, A_{2} \in R$, with assumption $b+h \neq d$. According to equation (6) we obtain

$$
A_{2}=-\frac{I_{0}+\Delta I}{1-(b+h) d^{-1}}
$$

and we have that

$$
Y_{2}^{*}(t)=-\frac{I_{0}+\Delta I}{1-(b+h) d^{-1}} d^{t} .
$$

Now we can establish the general solution of (4)

$$
Y(t)=A(b+h)^{t}+\frac{a+I_{0}+\Delta I}{1-b-h}-\frac{I_{0}+\Delta I}{1-(b+h) d^{-1}} d^{t}, \mathrm{t} \geq 0 .
$$

Hence it follows that the model (4) or (2) will be stable if $b+h<1$, i. e.

$$
h<1-b
$$

Since $0<d<1$, then every solution of (4) will converge to the value

$$
\frac{a+I_{0}+\Delta I}{1-b-h} .
$$

Since $1-b$ is the marginal propensity to save, condition (8) says that the marginal propensity to invest must be smaller than the marginal propensity to save if the model is to be stable.

Now, we will briefly investigate the case when $d=1$. From (4) we get

$$
Y(t)-(b+h) Y(t-1)=a .
$$

We treat this equation in a similar way as above. We obtain the particular solution $Y^{*}(t)$ of (9) under condition $b+h \neq 1$,

$$
Y^{*}(t)=\frac{a}{1-b-h},
$$

and also the general solution of (9)

$$
Y(t)=A(b+h)^{t}+\frac{a}{1-b-h} \cdot t \geq 0, A \in R .
$$

So, we have that the equilibrium of (9)

$$
\frac{a}{1-b-h}
$$

is stable if $b+h<1$.

\section{A model of the price of commodity}

By observation of the price $p(n)$ of a certain commodity in the period $n$ and number of units $D(n)$ demanded in period $n$, we obtained the following values

$$
\text { (p(1), } D(1)),(p(2), D(2)), \ldots(p(m), D(m)) \text {. }
$$

Using for example Gauss method of the least squares on the values above, we get the demand function $D(n)$. For simplicity of consideration we assume that $D(n)$ depends only linearly on $p(n)$ and has the concrete form

$$
D(n)=-p(n)+2.225, p \in[0.5,2.225] .
$$

Similarly let $S(n+1)$ be the number of units supplied in period $n+1$ with the price $p(n)$ one period before and we obtained the quantities

$$
(p / 1), S(2)), \ldots,(p(m), S(m+1)) .
$$

Using the method of least squares let the supplied function be

$S(n+1)=0.5 p^{2}(n)-0.5 p(n)+0.125, p \in[0.5,2.225]$.

We remind that the market price is the price at which the quantity demanded and the quantity supplied are equal, that is when

$D(n+1)=S(n+1)$.

By virtue of this fact we get

$p(n+1)=-0.5 p^{2}(2)-0.5 p(n)+2.1, p \in[0.5,2.225] .(10)$

The difference equation (10) is nonlinear and represents the model of market price. Generally we do not know how to solve such equations. But we can obtain important information about their dynamics, for example by equilibrium points, periodic points, cycles, etc.

We consider the difference equation

$$
x(n+1)=f(x(n)), n \geq 0 .
$$


Number $b \in R$ is called a periodic point with period $m \in N$ (i. e. $m$-periodic point) of continuous function $f: R \rightarrow R$ or equation (11), if $f^{m}(b)=b$ and $f^{j}(b) \neq b, j \in\{1, \ldots, m-1\}$,

where $f^{m}$ is the $m$ th iterate of function $f$.

Number $b \in R$ defines an $m$-cycle $\left\{b, f(b), \ldots, f^{m-1}(b)\right\}$ of function $f$ or equation (11), where

$$
f^{m}(b)=b .
$$

According to (10) we denote

$$
f(p)=-0.5 p^{2}+0.5 p+2.1 .
$$

Then the equilibrium points of Eq. (10) are obtained from the equation

$$
f(p)=p
$$

and we have $p_{1}=1.6095, p_{2}=-2.6095$. The point $p_{2} \notin[0.5$, 2.225 ] and the point $p_{1}$ represents the market price.

2-periodic points of (10) are obtained from the equation

$$
f^{2}(p)=p
$$

and we get $p_{3}=0.82918, p_{4}=2.17082$.

With regard to 2-periodic points the next 2-cycles of (10) follow

$$
\begin{aligned}
& \left\{p_{3}, f\left(p_{3}\right)\right\}=\{0.82918,2.17082\}, \\
& \left\{p_{4}, f\left(p_{4}\right)\right\}=\{2.17082,0.82918\} .
\end{aligned}
$$

From the economic point of view the 2-cycle of (10) envisages two prices of commodity: the low price $p_{3}=0.82918$ which is advantageous for a consumer and disadvantageous for a producer and high price $f\left(p_{3}\right)=p_{4}=2.17082$ suitable for a producer and unsuitable for a consumer. The cycle is depicted in Fig. 1.

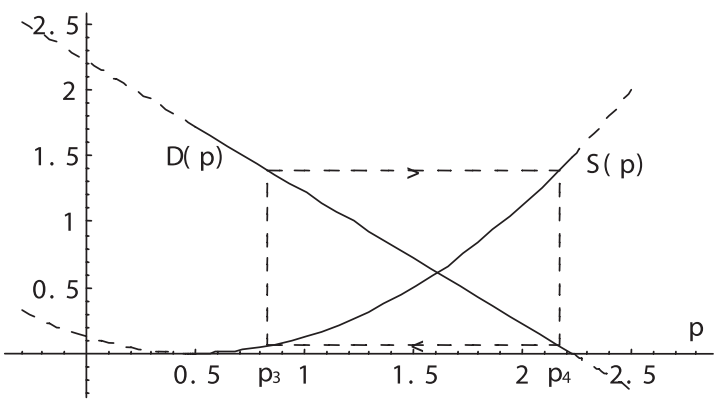

Fig. 1. 2-cycle of Eq. (10)

The pricing cycle is demonstrated in the market in such a way that due to the price $p_{3}$ there is a high demand $D\left(p_{3}\right)=1.39582$ and a low supply $S\left(p_{3}\right)=0.0541797$ and due to the price $p_{4}$ there is a low demand $D\left(p_{4}\right)=0.0541797$ and a high supply $S\left(p_{4}\right)=1.39582$.

We remind that 2-cycle $\left\{p_{3}, p_{4}\right\}$ is a periodic solution of the difference equation (10) and is depicted in Fig. 2.

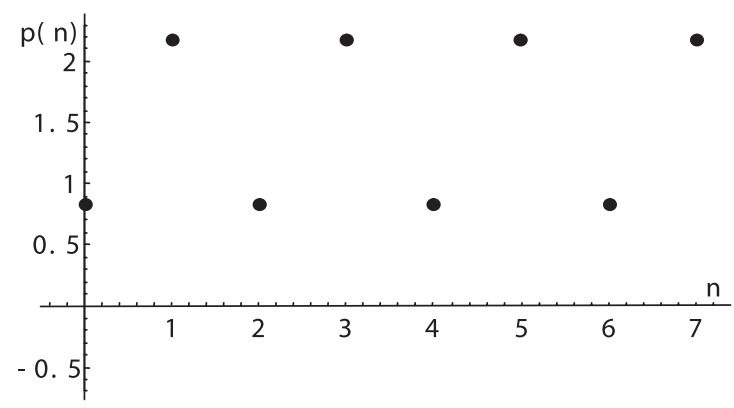

Fig. 2. Periodic solution of Eq. (10)

Observing the model (10) we get the necessary condition for existence of 2-cycle.

Theorem 1. If $\{d, h\}$ is 2-cycle of difference equation

$x(n+1)=a x^{2}(n)+b x(n)+c, a, b, c \in R, n \geq 0$,

then

$a(d+h)+b+1=0$.

Proof. Since $\{d, h]$ is 2-cycle and $f(x)=a x^{2}+b x+c$, we get $a d^{2}+b d+c=h, a h^{2}+b h+c=d$.

By subtraction we obtain

$a\left(d^{2}-h^{2}\right)+b(d-h)=h-d$,

$a(d+h)(d-h)+b(d-h)=h-d$,

$a(d+h)+b+1=0$.

The proof of theorem is complete.

\section{The stability of market price, periodic points and cycles}

The following theorems are our main tools in this section.

Theorem 2. Let $x^{*}$ be an equilibrium point of the difference equation (11), where $f$ is continuously differentiable at $x^{*}$. The following statements then hold true:

(i) if $\left|f^{\prime}\left(x^{*}\right)\right|<1$, then $x^{*}$ is an asymptotically stable (attracting) point,

(ii) if $\left|f^{\prime}\left(x^{*}\right)\right|>1$, then $x^{*}$ is not stable. In fact $x^{*}$ is a repelling point. 
The proof of Theorem 2 can be found in [2].

For the study of the stability of an $m$-periodic point $b$ of Eq. (11), one can use the previous Theorem 2 applied on $f^{m}$.

Theorem 3. Let $C(b)=\{b=x(0), x(1), \ldots, x(m-1)\}$ be an $m$-cycle of a continuously differentiable function $f$ or Eq. (11). Then the following statements hold:

(i) the $m$-cycle $C(b)$ is attracting if

$$
\left|f^{\prime}(x(0)) f^{\prime}(x(1)) \ldots f^{\prime}(x(m-1))\right|<1,
$$

(ii) the $m$-cycle $C(b)$ is repelling if

$$
\left|f^{\prime}(x(0)) f^{\prime}(x(1)) \ldots f^{\prime}(x(m-1))\right|>1 .
$$

The proof of Theorem 3 is in [2].

Using Theorem 2 for $p_{1}=1.6095$ we get

$$
\left|f^{\prime}\left(p_{1}\right)\right|=|-1.1095|=1.1095 \text {. }
$$

That is the market price $p_{1}$ of the model (10) is not stable.

Now we will study the stability of 2-periodic points $p_{3}$ and $p_{4}$ For $p_{3}=0.82918$ and $p_{4}=2.17082$ we get

$$
\left|\left(f^{2}\right)^{\prime}\left(p_{3}\right)\right|=0.55,\left|\left(f^{2}\right)^{\prime}\left(p_{4}\right)\right|=0.55 .
$$

Both periodic points are asymptotically stable.
By virtue of Theorem 3 for cycles $\left\{p_{3}, f\left(p_{3}\right)\right\}$ and $\left\{p_{4}, f\left(p_{4}\right)\right\}$ we obtain

$$
\begin{aligned}
& \left|f^{\prime}\left(p_{3}\right) f^{\prime}\left(f\left(p_{3}\right)\right)\right|=|-0.32918(-1.67082)|=0.55, \\
& \left|f^{\prime}\left(p_{4}\right) f^{\prime}\left(f\left(p_{4}\right)\right)\right|=|-1.67082(-0.32918)|=0.55 .
\end{aligned}
$$

So we have that both cycles are asymptotically stable. Since the cycle $\{0.82918,2.17082\}$ is attracting (asymptotically stable), every solution of (10) converges to its periodic solution.

\section{Conclusions}

We can conclude that the model (4) of the national income will be stable, if the marginal propensity to invest is smaller than the marginal propensity to save.

From the economic point of view the model (10) interprets a very common situation in the market at which the price of commodity satisfies the consumer and does not satisfy the producer and vice versa. In this case it is suitable to change the supply and demand function or to come to the market with a new commodity.

\section{Acknowledgements}

The research was partially supported by the grant $1 / 0026 / 03$ of the Scientific Grant Agency of Ministry of Education of the Slovak Republic and Slovak Academy of Sciences.

\section{References}

[1] BAČOVÁ, B., DOROCIAKOVÁ, B., OLACH, R.: Oscillations in Epidemic Model, Studies of University in Žilina, Vol. 13, 2001, pp. 17-24.

[2] ELAYDI, S. N.: An Introduction to Difference Equations, Springer-Verlag, New York-Berlin-London-Paris-Tokyo, 1996.

[3] GANDOLFO, G.: Economic Dynamics, Springer-Verlag, Berlin-New York-London-Tokyo, 1997.

[4] OLACH, R., SMEJOVÁ, S., ŠVAŇOVÁ, M.: On the Neoclassical Growth Model, $4^{\text {th }}$ Int. Confer. APLIMAT 2005, Bratislava, pp. $105-110$.

[5] VÁCLAVÍKOVÁ, B.: Dynamic Model of an Economic Process, $4^{\text {th }}$ Int. Confer. APLIMAT 2005, Bratislava, pp. 167-172. 